\title{
Active Disturbance Rejection Control of a SCARA Robot Arm
}

\author{
Ali Medjebouri ${ }^{1}$ and Lamine Mehennaoui ${ }^{2}$ \\ ${ }^{1}$ Department of Mechanical Engineering, Skikda University, Algeria \\ ${ }^{2}$ Department of Electrical Engineering, Skikda University, Algeria \\ ${ }^{1}$ ali.medjbouri@gmail.com, ${ }^{2}$ me_lamine@yahoo.fr
}

\begin{abstract}
This paper is focused on a comparison between classic Feedback Linearization Control and a proposed Active Disturbance Rejection Control method. The proposed technique does not require a precise mathematical description of the system, since it is based on the online estimation and rejection of the unmodeled elements of the dynamics. Robustness of the closed-loop control system (against external perturbation and parameters uncertainty) is discussed here. A model of a SCARA robot manipulator is used in the conducted case study as an exemplary plant. Conclusions are supported with results obtained with numerical simulations.
\end{abstract}

Keywords: SCARA, Position Control, Feedback Linearization Control, Active Disturbance Rejection Control, Tracking Performances, Robustness

\section{Introduction}

The SCARA (selectively compliant assembly robot arm) robot arm, Figure 1, uses two parallel revolute joints to produce motion in the horizontal plane. The arm structure is weight-bearing but the first and second axes do no lifting. The third axis of the SCARA provides work volume. By adding a fourth revolute joint, an additional rotation about the $\mathrm{Z}$ axis is added to control the orientation of the end effector in the horizontal plane. This type of robots is rarely found with more than four axes. The SCARA robot manipulators are used extensively in the assembly of electronic components and of small and medium sized mechanical devices [1-3].

In controlling, the robot arm head is moved by actuators to attain an exact moving, and the dynamic model of the robot manipulator is a MIMO (Multiple-Input Multiple-Output) highly non-linear coupled system [2,3].

Nonlinear systems control has been widely concern of the research. At present, the nonlinear system decoupling control and static feedback linearization that based on the theory of differential geometry brought the research getting rid of limitation for local linearization and small scale motion [4]. However, differential geometry control must depend on precise mathematical model, and when system works with various payloads and has uncertainty in dynamic model this technique has limitations [5]. From control point of view, uncertainty is divided into two main groups: uncertainty in unstructured inputs (e.g., noise, external disturbance, and unmodeled dynamics) and uncertainty in structure dynamics (parametric uncertainties). In some applications systems are used in an unknown and unstructured environment, therefore strong mathematical tools are used in new control methodologies to design nonlinear robust controllers with acceptable performances (e.g., minimum error, good trajectory tracking, and disturbance rejection) [6].

One ideal candidate is the Active Disturbance Rejection Control strategy (ADRC) which has a very good disturbance rejection capability. The ADRC was firstly proposed by Han in 1990s [7, 8] and further simplified and explicated by Gao in [9, 10]. The essence of ADRC is that both the internal dynamics and the external disturbances can be 
estimated and compensated in real time [6-8]. Due to its strong robustness and disturbance rejection, ADRC has been successfully applied in many fields $[7,8,11]$.

In this paper, we propose to improve classic feedback linearization control robustness by introducing an active disturbance rejection component. The proposed strategy will be applied for the position control of an electrically driven two-link SCARA robot arm.

(a)

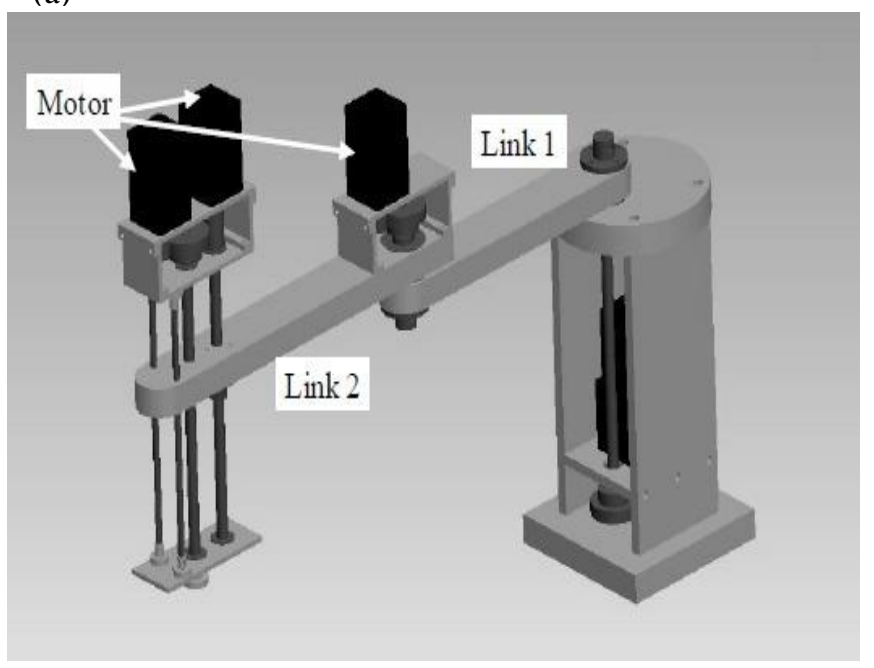

(b)

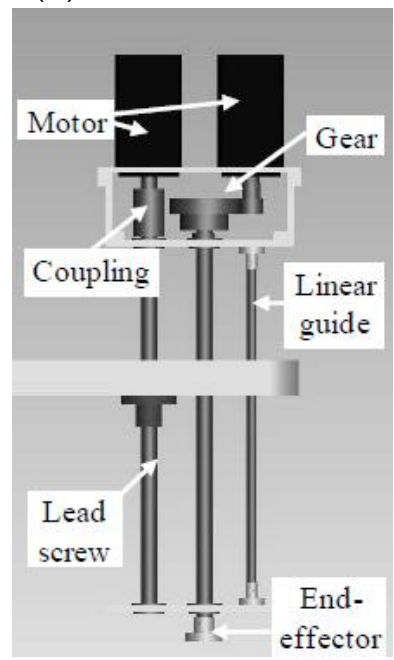

Figure 1.SCARA robot arm design: (a). full manipulator.(b). End effector.

\section{SCARA Horizontal Plane Dynamics Modeling}

The SCARA robot uses the first two joints to move around in the horizontal plane. Only these axes are dynamically coupled. The third and fourth axis can be considered as decoupled mechanically from the motions of the other axes.

The manipulator powered by DC motors is modeled by the following dynamic expression $[1,12]$ :

$$
\left\{\begin{array}{c}
M(q) \cdot \ddot{q}+C(q, \dot{q})+F(q, \dot{q})+G(q)=K_{T} I \\
L_{a} \dot{I}+R_{a} I+K_{e} N \dot{q}=V
\end{array}\right.
$$

Where symbols $q, \dot{q}$, and $\ddot{q}$ denote two-dimensional vectors of joint position, angular velocity and angular acceleration. $M(q) \in R^{2 \times 2}$ is a symmetric, positive definite inertia and mass matrix. $C(q, \dot{q}) \in R^{2 \times 1}$ is the matrix of the Coriolis and centrifugal forces. $G(q) \in R^{2 \times 1}$ is the vector of gravity terms, and $F(q, \dot{q}) \in R^{2 \times 1}$ is the friction torques vector considered as unknown external disturbances.

$I \in R^{2 \times 1}$ is the armature current, $V \in R^{2 \times 1}$ is the armature voltage, and $L_{a}, R_{a}, K_{e}, K_{T}, N$ are positive definite diagonal matrices, representing respectively, the actuators inductances, the actuators resistances, back electromotive force coefficients, the constant coefficients characterizing the electromechanical conversion between current and torque, and gearboxes ratios.

Since the first two axes only move the robot in the horizontal plane, the Gravity vector is zero. The inertia, Coriolis and friction torques matrices for the proposed SCARA robot arm are as follow [13]: 


$$
\begin{aligned}
M_{11}=J_{m 1}+ & \frac{\left(J_{1}+J_{2}\right)}{N_{1}^{2}}+\frac{m_{1} r_{1}^{2}+m_{2} r_{2}^{2}+4 m_{2} r_{1}^{2}}{4 N_{1}^{2}} \\
& +\frac{m_{2} r_{1} r_{2}}{N_{1}^{2}}\left(\cos \left(q_{1}+q_{2}\right) \cos \left(q_{1}\right)+\sin \left(q_{1}+q_{2}\right) \sin \left(q_{1}\right)\right) \\
M_{12}= & \frac{J_{2}}{N_{1} N_{2}}+\frac{m_{2} r_{2}^{2}}{4 N_{1} N_{2}}+\frac{m_{2} r_{1} r_{2}}{2 N_{1} N_{2}}\left(\cos \left(q_{1}+q_{2}\right) \cos \left(q_{1}\right)+\sin \left(q_{1}+q_{2}\right) \sin \left(q_{1}\right)\right) \\
M_{21}= & \frac{J_{2}}{N_{1} N_{2}}+\frac{m_{2} r_{2}^{2}}{4 N_{1} N_{2}}+\frac{m_{2} r_{1} r_{2}}{2 N_{1} N_{2}}\left(\cos \left(q_{1}+q_{2}\right) \cos \left(q_{1}\right)+\sin \left(q_{1}+q_{2}\right) \sin \left(q_{1}\right)\right) \\
M_{22}= & J_{m 2}+\frac{J_{2}}{N_{2}^{2}}+\frac{m_{2} r_{2}^{2}}{4 N_{2}^{2}} \\
C_{11}= & \frac{m_{2} r_{1} r_{2}}{N_{1}}\left(\dot{q}_{1} \cdot \dot{q}_{2}+\frac{\dot{q}_{2}^{2}}{2}\right)\left(\cos \left(q_{1}+q_{2}\right) \sin \left(q_{1}\right)-\sin \left(q_{1}+q_{2}\right) \cos \left(q_{1}\right)\right) \\
C_{21}= & \frac{m_{2} r_{1} r_{2}}{2 N_{2}}\left(\dot{q}_{1}^{2}\right)\left(\sin \left(q_{1}+q_{2}\right) \cos \left(q_{1}\right)-\cos \left(q_{1}+q_{2}\right) \sin \left(q_{1}\right)\right) \\
F_{11}= & f_{c 1} \operatorname{sign}\left(\dot{q}_{1}\right)+f_{v 1} \dot{q}_{1} \\
F_{21}= & f_{c 2} \operatorname{sign}\left(\dot{q}_{2}\right)+f_{v 2} \dot{q}_{2}
\end{aligned}
$$

In order to facilitate the control task, we propose to simplify (1) by neglecting the armature inductance. Then current expression becomes:

$$
I=R_{a}^{-1} \cdot\left[V-K_{e} N \dot{q}\right]
$$

Finally, system (1) is reduced to:

$$
M(q) . \ddot{q}+h(q, \dot{q})=K . V
$$

Where:

$$
\left\{\begin{array}{c}
h(q, \dot{q})=C(q, \dot{q})+F(q, \dot{q})+K_{T} \cdot R_{a}^{-1} \cdot K_{e} N \dot{q} \\
K=K_{T} \cdot R_{a}^{-1}
\end{array}\right.
$$

\section{Active Disturbance Rejection Control of the Robot Manipulator}

Consider first the dynamic system given by the expression (3); the design of the proposed controller is based on the following static nonlinear state feedback control, where $v$ is the new control vector:

$$
K . V=M(q) \cdot v+h(q, \dot{q})
$$

If $M$ is nonsingular, this control input simplifies (3) to the following linearized decoupled system:

$$
\ddot{q}=v
$$

Taking into account the uncertainty of the system model, we can then express the nonlinear control law (4) as follows:

$$
K . V=\widehat{M}(q) \cdot v+\widehat{h}(q, \dot{q})
$$

Where $\widehat{M}, \hat{h}$ are the nominal values of $M, h$. The model errors are then represented as: 


$$
\left\{\begin{array}{c}
\Delta M=\widehat{M}(q)-M(q) \\
\Delta h=\widehat{h}(q)-h(q)
\end{array}\right.
$$

From (1) and (6), we find:

$$
\widehat{M}(q) \cdot v+\widehat{h}(q, \dot{q})=M(q) \cdot \ddot{q}+h(q, \dot{q})
$$

This can be expressed as follows:

$$
\begin{aligned}
\ddot{q} & =M^{-1} \cdot \widehat{M} \cdot v+M^{-1} \cdot \Delta h \\
& =v+\left(M^{-1} \cdot \widehat{M}-I\right) \cdot v+M^{-1} \cdot \Delta h
\end{aligned}
$$

We then define:

$$
\left\{\begin{array}{c}
E=\left(M^{-1} \cdot \widehat{M}-I\right) \\
\eta=E \cdot v+M^{-1} \cdot \Delta h
\end{array}\right.
$$

Substituting (9) into (8), we obtain finally the effect of disturbances on the linearized model:

$$
\ddot{q}=v+\eta(v, q, \dot{q})
$$

In our case, the system dynamics are given by:

$$
\left\{\begin{array}{l}
\ddot{q}_{1}=v_{1}+\eta_{1}(v, q, \dot{q}) \\
\ddot{q}_{2}=v_{2}+\eta_{2}(v, q, \dot{q})
\end{array}\right.
$$

And the control outputs are:

$$
\left\{\begin{array}{l}
y_{1}=q_{1} \\
y_{2}=q_{2}
\end{array}\right.
$$

Both systems (11) can be rewritten as follows:

$$
\left\{\begin{array}{c}
\dot{x}_{i 1}=x_{i 2} \\
\dot{x}_{i 2}=x_{i 3}+b . v_{i} \\
\dot{x}_{i 3}=\dot{\eta}_{l}(.)
\end{array}\right.
$$

Where:

$$
\left\{\begin{array}{c}
x_{i 1}=\left.q_{i}\right|_{i=1,2} \\
x_{i 2}=\left.\dot{q}_{i}\right|_{i=1,2} \\
x_{i 3}=\left.\eta_{i}(v, q, \dot{q})\right|_{i=1,2} \\
b=1
\end{array}\right.
$$

$\eta_{i}($.$) is an unknown function which can be viewed as the total uncertainties or$ disturbances of the system. Considering $\eta_{i}($.$) as an augmented state, it can be easily$ observed. In $[14,16]$ we find the following nonlinear extended state observer (ESO: Extended State Observer):

$$
\left\{\begin{array}{c}
\dot{\hat{x}}=A_{i} \cdot \hat{x}+B_{i} \cdot v_{i}+L_{i} \cdot f a l\left(e_{i}, \alpha_{i}, \delta_{i}\right) \\
y=C_{i} \hat{x}
\end{array}\right.
$$


Where:

$$
A_{i}=\left[\begin{array}{lll}
0 & 1 & 0 \\
0 & 0 & 1 \\
0 & 0 & 0
\end{array}\right], B_{i}=\left[\begin{array}{l}
0 \\
b \\
0
\end{array}\right], C_{i}=\left[\begin{array}{lll}
1 & 0 & 0
\end{array}\right], L_{i}=\left[\begin{array}{l}
L_{i 1} \\
L_{i 2} \\
L_{i 3}
\end{array}\right]
$$

The observer errors are:

$$
\left.e_{i}\right|_{i=1,2}=q_{i}-\hat{x}_{1}
$$

Functions $f a l_{i j}$ are defined as:

$$
\left.\operatorname{fal}_{i j}\left(e_{i}, \alpha_{i j}, \delta_{i}\right)\right|_{\substack{i=1,2 \\ j=1,2,3}}= \begin{cases}\left|e_{i}\right|^{\alpha_{i j}} \cdot \operatorname{sign}\left(e_{i}\right), & \text { if }\left|e_{i}\right|>\delta_{i} \\ \frac{e_{i}}{\delta_{i}{ }^{1-\alpha_{i j}}}, & \text { otherwise }\end{cases}
$$

Here $0<\alpha_{i j}<1$ is the nonlinear coefficient, $\delta_{i}>0$ is the turning point of $f a l$ function. Note that, if the error is small $\left(\left|e_{i}\right| \leq \delta_{i j}\right)$; the proposed ESO becomes a simple linear observer (LESO):

$$
\left\{\begin{array}{c}
\dot{x}_{i 1}=x_{i 2}+\beta_{i 1} e_{i} \\
\dot{x}_{i 2}=x_{i 3}+\beta_{i 2}+b v_{i} \\
\dot{x}_{i 3}=\beta_{i 3} e_{i}
\end{array}\right.
$$

Where:

$$
\beta_{i j}=\left.\frac{L_{i j}}{\delta_{i}{ }^{1-\alpha_{i j}}}\right|_{\substack{i=1,2 \\ j=1,2,3}}
$$

The parameters $\beta_{i j}$ are chosen in a special way as [7-9, 14]:

$$
s^{3}+\beta_{i 1} s^{2}+\beta_{i 2} s+\beta_{i 3}=\left.\left(s+\omega_{0 i}\right)^{3}\right|_{i=1,2}
$$

Where $\omega_{0 i}$ denotes the bandwidth of the LESO (17).

Once uncertainties and disturbances effects are totally estimated by the ESO, we can now compensate them in real time by the use of the control signal $v_{i}[8-10,14,15,17]$ :

$$
v_{i}=\left(v_{i 0}-\hat{x}_{i 3}\right) / b
$$

Appliying this control input, system (11) is reduced to:

$$
\ddot{q}_{i}=\left(x_{i 3}-\eta_{i}\right)+v_{i 0}
$$

When $\hat{x}_{i 3} \rightarrow \eta_{i}$ :

$$
\ddot{q}_{i} \approx v_{i 0}
$$

The expression (21) represents a double integrator with unit gain, which can be readily driven to the desired position by the use of a simple linear controller. Let $q_{d i}$ denote the reference position for each joint, and the tracking errors $e_{i}=q_{d i}-q_{i}$. Then the linear controller can be defined as:

$$
v_{i 0}=\ddot{q}_{d i}+2 \zeta_{c i} \omega_{c i} \dot{e}_{l}+\omega_{c i}^{2} e_{i}
$$

Which leads to the following second order tracking dynamics in the closed loop:

$$
\ddot{e}_{i}+2 \zeta_{c i} \omega_{c i} \dot{e}_{\imath}+\omega_{c i}^{2} e_{i}=0
$$


Where $\zeta_{c i}, \omega_{c i}$ are respectively the damping ratio and the natural frequency of the closed-loop system. The bandwidth $\omega_{0 i}$ of the ESO is chosen to ensure a faster dynamics than (23):

$$
\omega_{0 i}=(3 \text { to } 5) \omega_{c i}
$$

The block diagram of the ADRC strategy is shown in Figure 2.

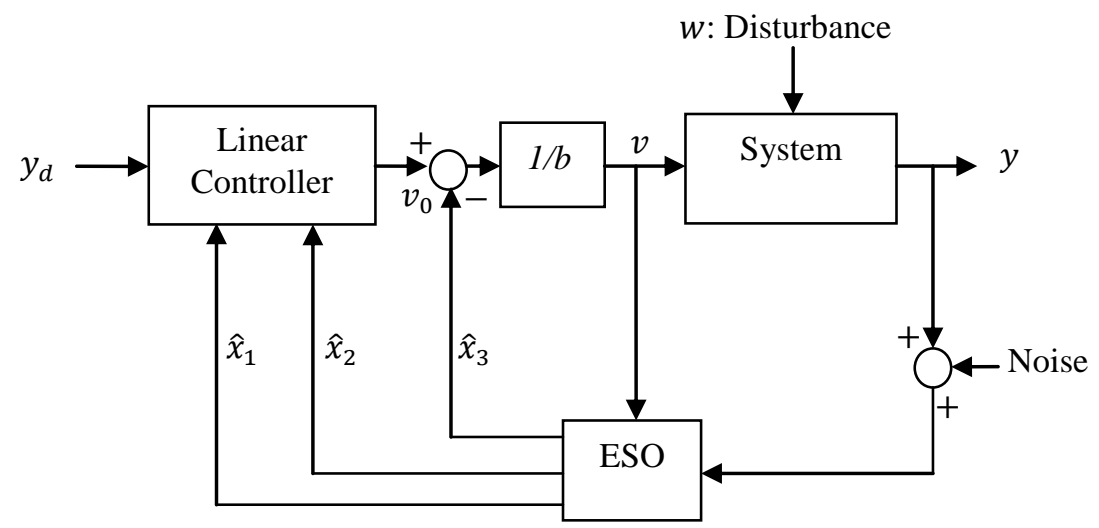

Figure 2. Block Diagram of the ADRC Control Strategy

Note that if the active rejection component $\hat{x}_{i 3}$ is not introduced in the control law (19), the proposed technique is reduced to the classical feedback linearization control method (FLC).

\section{Results and Discussion}

The above proposed control strategy for a two-link SCARA robot manipulator electrically controlled has been verified through computer simulations using Matlab/Simulink. The block diagram of the proposed control strategy is shown in the Figure 3, and the parameters of the considered robot manipulator are those indicated in the appendix.

For measuring the performances in both tracking (reaching time, accuracy) and regulation (disturbances rejection) modes we will consider two cases:

Case1(tracking performances measurement): the control law is applied on the nominal system withaout considering internal or external disturbaces effects (parameters uncertaintys and friction torques respectively). Since the active rejection terme is inactivated, the proposed control method is reduced to the classical feedback lineariszation control scheme (FLC).

Case2 (disturbances rejection measurement): friction torques considered as external disturbances and a wide parameters uncertainty $\Delta M=50 \% \widehat{M}(q), \Delta h=$ $50 \% \hat{h}(q)$ are introduced to the manipulator model, and the proposed technique with an active disturbance rejection control component (ADRC) is compared to the classical feedback linearization control strategy (FLC). 


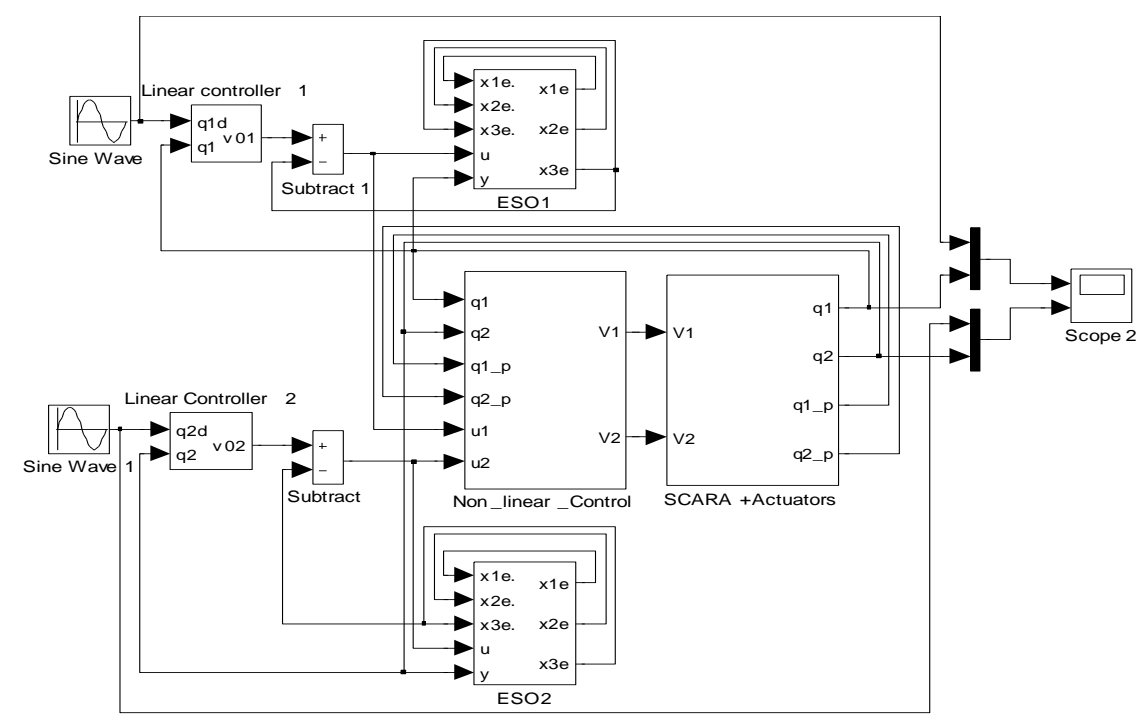

Figure 3. Block Diagram of the Proposed Control Strategy

The obtained results by the 4 th order Runge Kutta solver with a step size $\Delta t=0.001 \mathrm{sec}$ are given below:

\section{Case1:}

Link 1
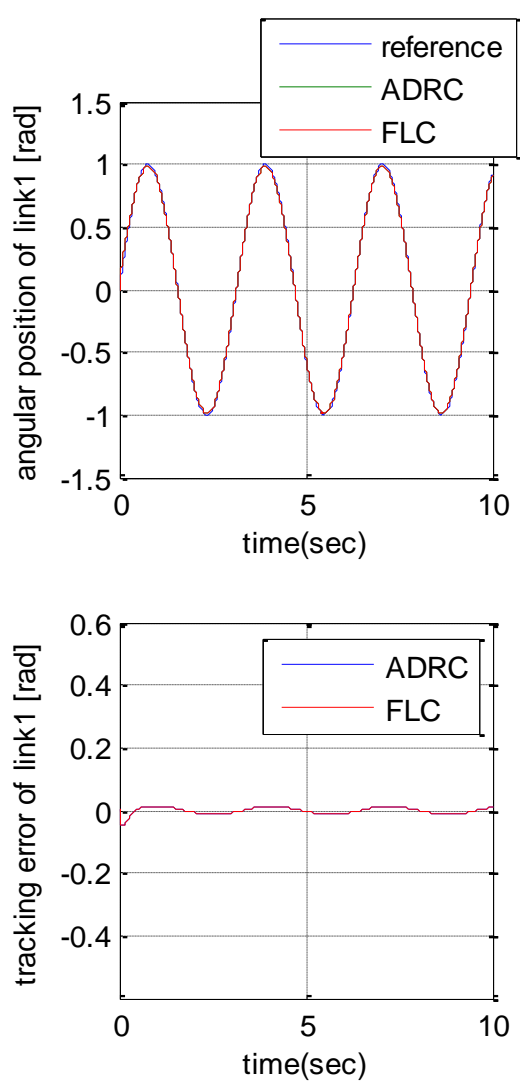

Link 2
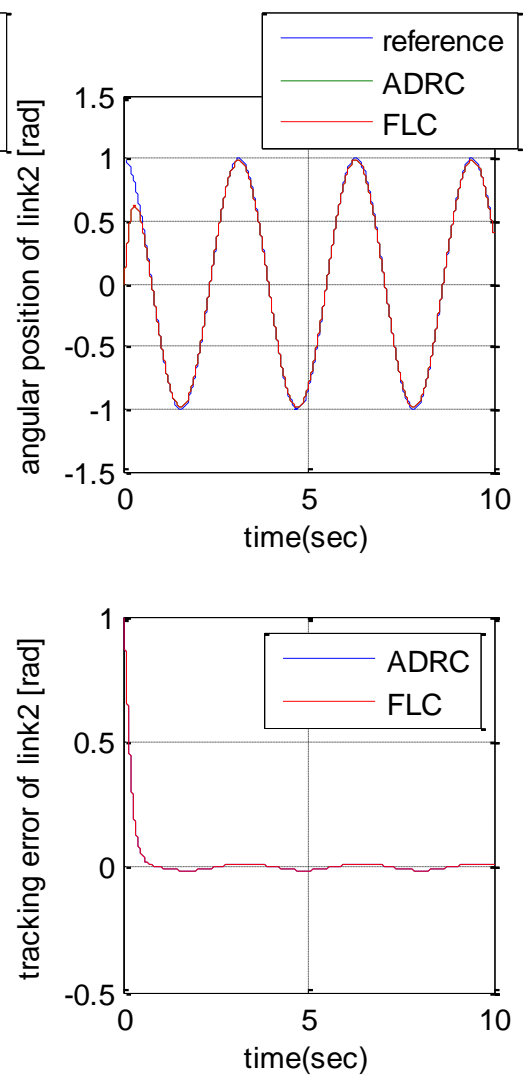

Figure 4. Tracking Performances 
Link 1

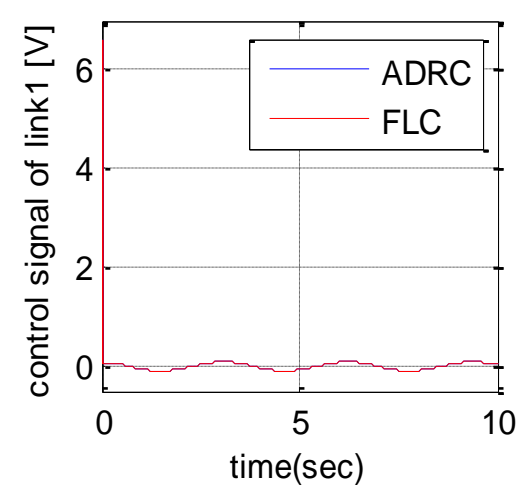

Figure 5. Control inputs
Link 2

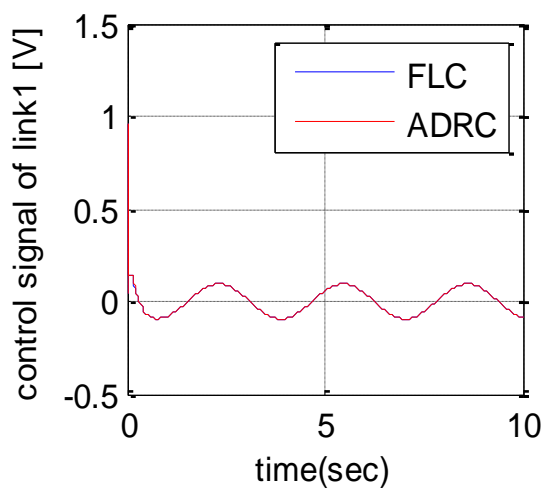

Link 1
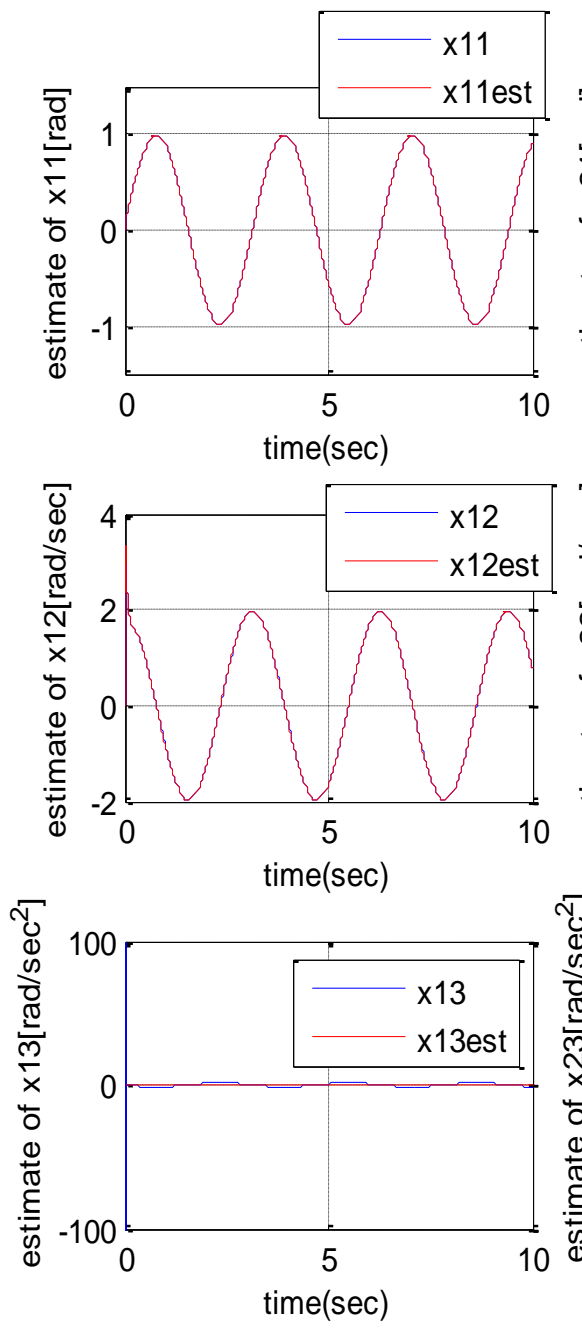

Figure 6. ESO Estimated States Without Disturbances
Link 2
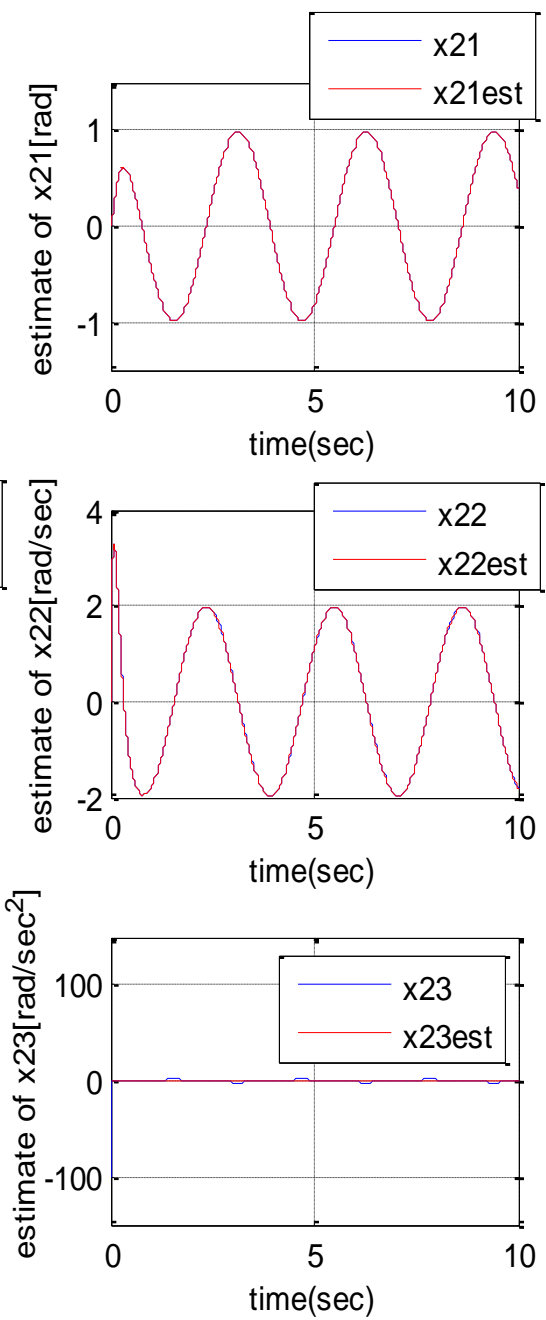

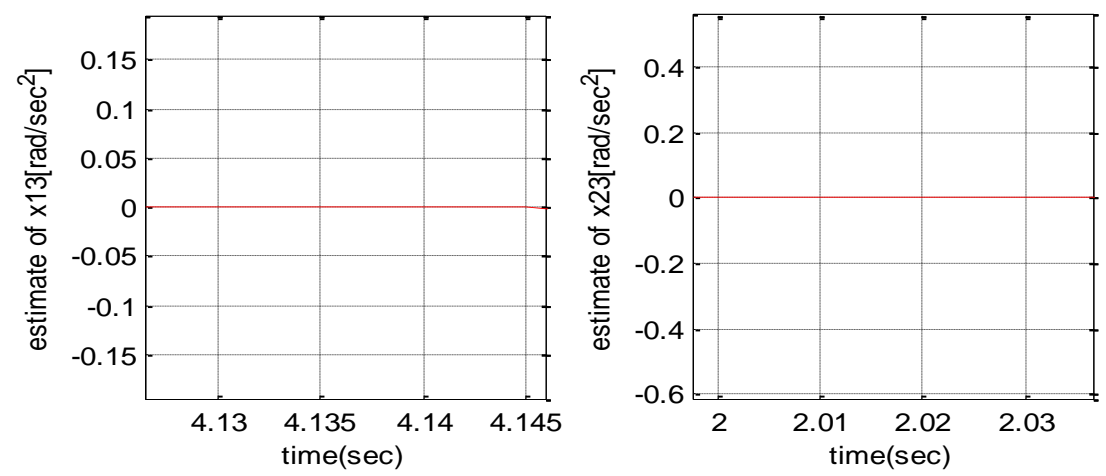

Figure 7. Zoom on $x_{13}$ and $x_{23}$ Estimated States

\section{Case 2:}

Link 1
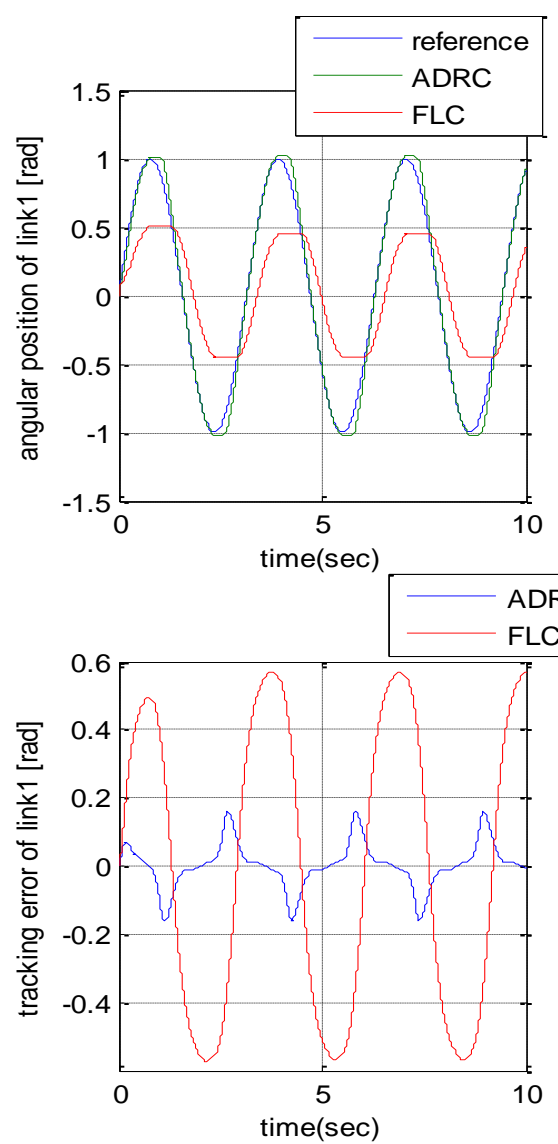

Figure 8. Tracking performances in Presence of Disturbances

\section{Link 2}
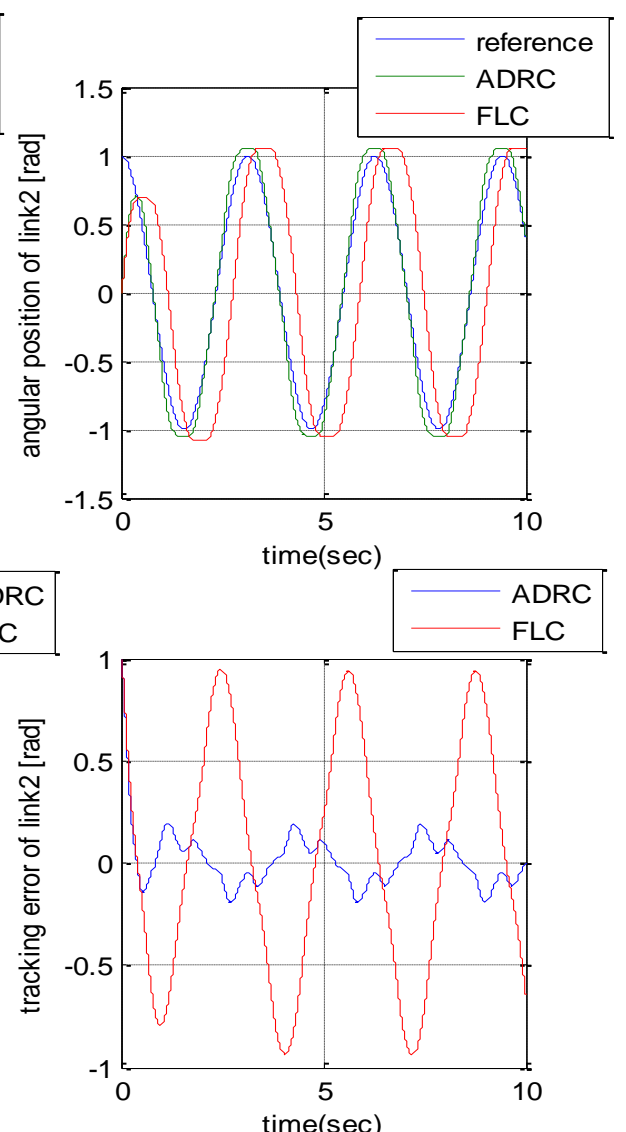
Link 1

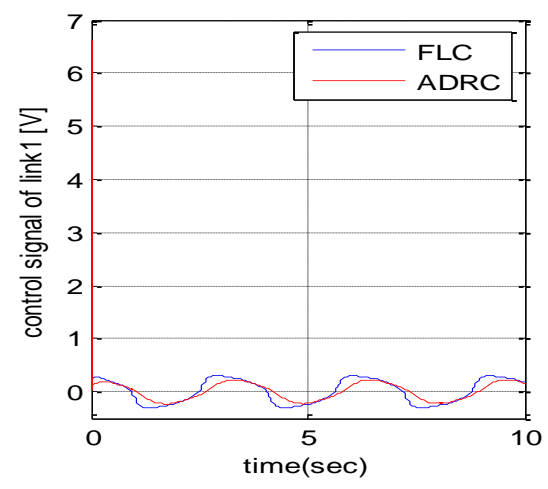

Link 2

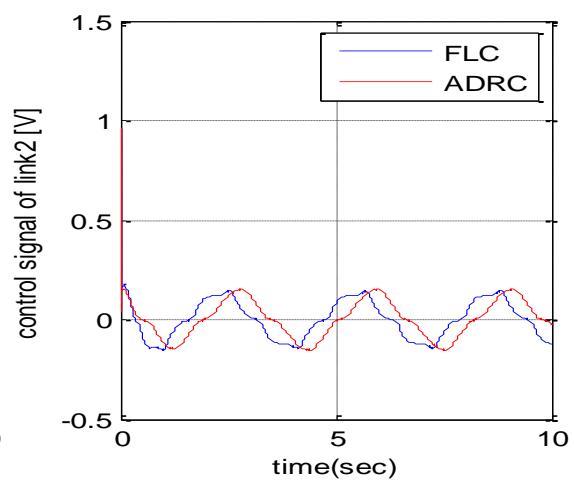

Figure 9. Control Inputs in Presence of Disturbances

Link1
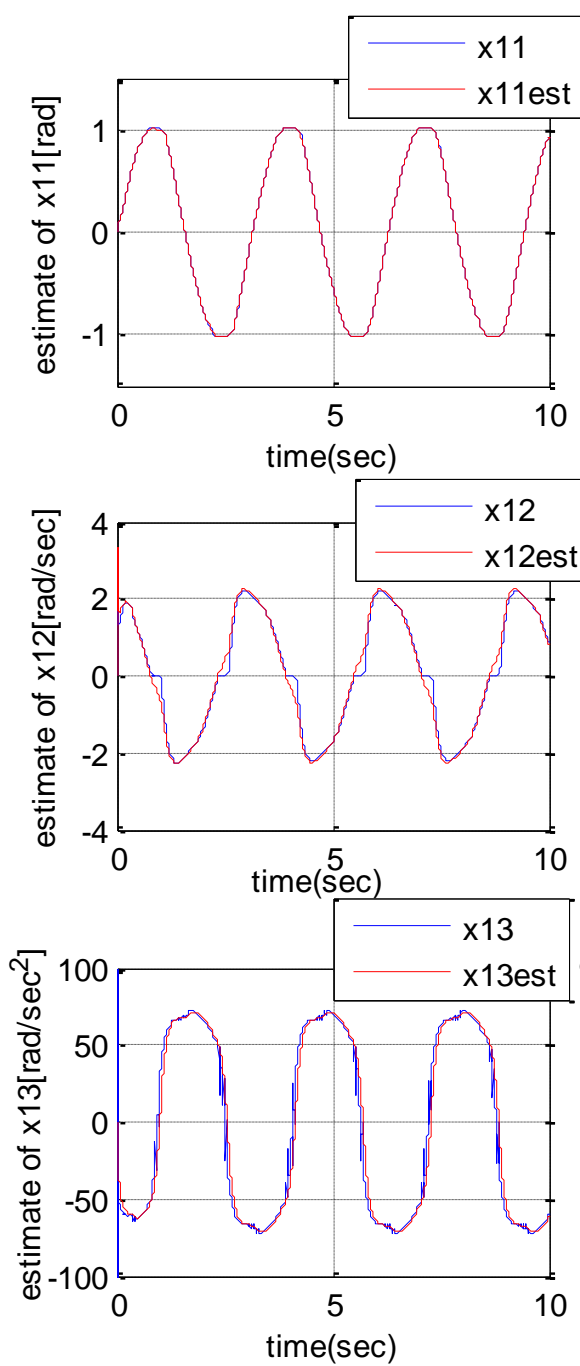

Link2
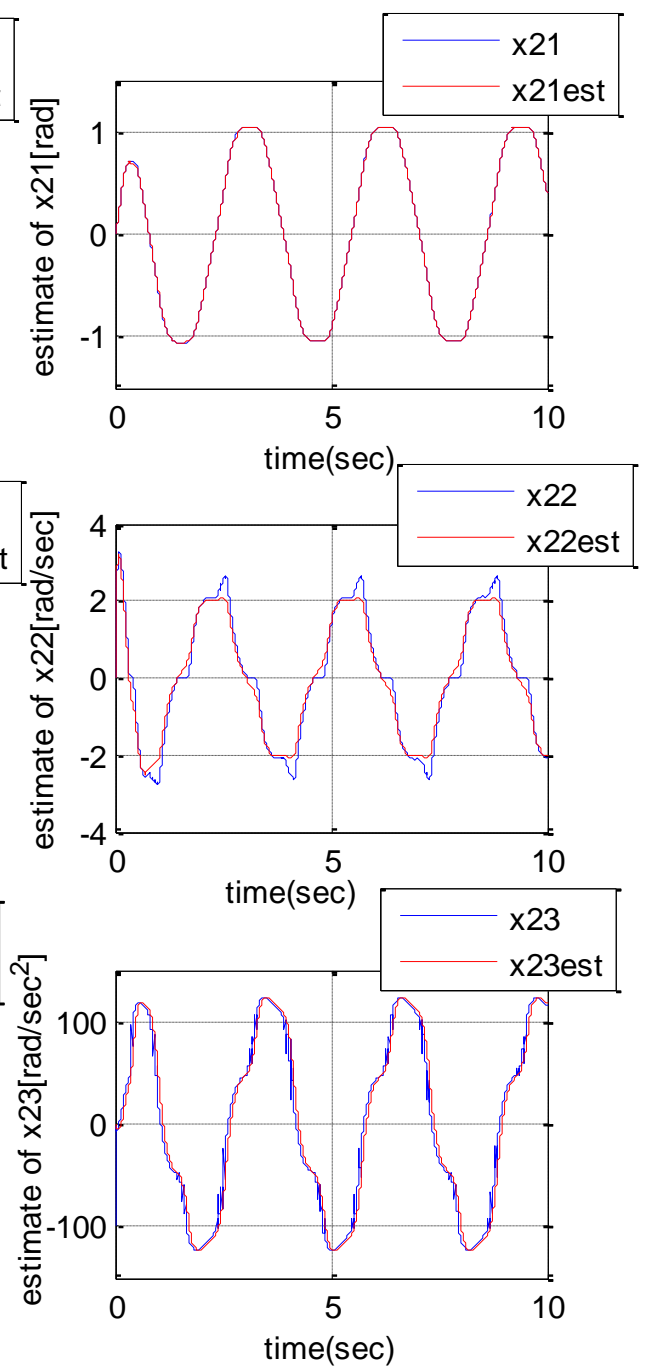

Figure 10. ESO Estimated States in Presence of Disturbances 
Case1: Since the system is not subject to disturbances effects, and as provided both strategys give exactly the same good results in tracking mode, Figure 4. The Figure 6 shows clearly the convergence of the observed states to the real system states.

Case2: The simulation results presented in the Figure 8 highlight the effectiveness and the superiority of the proposed ADRC control strategy compared with classical feedback control law (FLC) in presence of wide parametric and external disturbances. This means that the extended state observer was able to observe and decrease disturbances effects which is confirmed in Figure 10. The Figure 9 shows the control inputs and the compensation components effects.

\section{Conclusion}

In this paper, active disturbance rejection control method (ADRC) was successfully applied to the position control of a two-link SCARA robot arm electrically actuated. Simulation results demonstrate that the proposed control algorithm can provide good tracking performance and well handle disturbances for the robot manipulator.

It should be noted that the design of this control strategy does not require a high accuracy mathematical model of the controlled system. This practical control strategy is easy to understand and implement, making it an appealing method to real applications.

\section{Appendix:}

Parameters of the robot arm:

$m_{1}=1,90 \mathrm{~kg}, m_{2}=0,93 \mathrm{~kg}, J_{1}=0,0980 \mathrm{kgm}^{2}, J_{2}=0,0115 \mathrm{kgm}^{2}, J_{m 1}=J_{m 2}=$ $3,3 \cdot 10^{-6} \mathrm{kgm}^{2}, r_{1}=0,25 \mathrm{~m}, r_{2}=0,15 \mathrm{~m}, R_{a 1}=R_{a 2}=3,5 \Omega, L_{a 1}=L_{a 2}=1,3 \mathrm{mH}$, $K_{e 1}=K_{e 2}=0,047 \mathrm{~V} / \mathrm{rad} / \mathrm{s}, K_{T 1}=K_{T 2}=0,047 \mathrm{Nm} / \mathrm{A}, N_{1}=90, N_{2}=220$.

Parameters of the extended state observer:

$\alpha_{i j}=0,5 ; \quad \delta_{i}=0,01 ; \omega_{0 i}=5 \omega_{c i} \quad$ for $i=\overline{1,2}$ and $j=\overline{1,3}$.

Parameters of the linear controller:

$\zeta_{c i}=1 ; \quad \omega_{c i}=10 \mathrm{rad} / \mathrm{sec}$ for $i=\overline{1,2}$

\section{References}

[1] N. Munro and F. L. Lewis, Robot Manipulator Control-Theory and Practice, 2nd edition, Marcel Dekker Inc., (2004).

[2] A. A. Amiri-M, M. R. Gharib, M. Moavenian and K. Torabi Z. Modeling and control of a SCARA robot using quantitative feedback theory. Journal of Systems and Control Engineering. Part I, 223 (2009).

[3] K. Sahari, K. H. Weng, Y. W. Han, A. Anuar, M. Z. Baharuddin and S. Mohideen. Design and Development of a 4-DOF SCARA Robot for Educational Purposes. Jurnal Teknologi, Universiti Teknologi Malaysia. (2011).

[4] C. Kravaris and C. B. Chung. Nonlinear State Feedback Synthesis by Global Input /Output Linearization. AIChE Journal. 4, 33 (1987).

[5] C. Kravaris and S. Palanki. Robust Nonlinear State Feedback under Structured Uncertainty. AIChE Journal. 7, 34 (1988).

[6] F. Piltan, M. R. Rashidian, M. Shamsodini and S. Allahdadi. Effect of Rule Base on the Fuzzy-Based Tuning Fuzzy Sliding Mode Controller: Applied to 2nd Order Nonlinear System. International Journal of Advanced Science and Technology. 46 (2012).

[7] S. Liu, X. Mei and F. Kong. Research on Unwinding Tension System Control Based on Active Disturbance Rejection Control. 2nd International Conference on Materials, Mechatronics and Automation, (2012) May 7-8, 2012, Nanchang, China, pp. 252-257.

[8] J. Zhang, J. Feng, Y. Zhou, F. Fang and H. Yue. Linear Active Disturbance Rejection Control of Waste Heat Recovery Systems with Organic Rankine Cycles. Energies. 5 (2012).

[9] Z. Gao. Active Disturbance Rejection Control: A Paradigm Shift in Feedback Control System Design. Proceedings of the 2006 American Control Conference, (2006) June 14-16; Minnesota, USA, pp. 23992405. 
[10] Q. Zheng, L. Q. Gao and Z. Gao. On Stability Analysis of Active Disturbance Rejection Control for Nonlinear Time-Varying Plants with Unknown Dynamics. Proceedings of the 46th IEEE Conference on Decision and Control. (2007) December 12-14; New Orleans, USA, pp. 3501-3506.

[11] Q. Zheng and Z. Gao. On Practical Applications of Active Disturbance Rejection Control. Proceedings of the 29th Chinese Control Conference. (2010) July 29-31; Beijing, China, pp. 6095-610.

[12] N. Azoui and L. Saidi. Passivity Based Adaptive Control of Robotic Manipulators Electrically Controlled. International Journal of Advanced Science and Technology. 34 (2011).

[13] M. T. Das and L. C. Dülger. Mathematical Modeling, Simulation and Experimental Verification of a SCARA Robot. Simulation Modelling Practice and Theory. 13 (2005).

[14] J. Han. From PID to Active Disturbance Rejection Control. IEEE Transactions on Industrial Electronics. 3, 56 (2009).

[15] Q. Zheng, L. Q. Gao and Z. Gao. On Estimation of Plant Dynamics and Disturbance from Input-Output Data in Real Time. 16th IEEE International Conference on Control Applications. (2007) October 1-3; Singapore, pp.1167-1172.

[16] X. Yang and Y. Huang. Capabilities of Extended State Observer for Estimating Uncertainties. 2009 American Control Conference. (2009) June 10-12; St. Louis, USA, pp. 3700-3705.

[17] A. Boukhriss, T. Nasser and A. Essadki. A Linear Active Disturbance Rejection Control applied for DFIG based Wind Energy Conversion System. International Journal of Computer Science Issues. 2, 10 (2013)

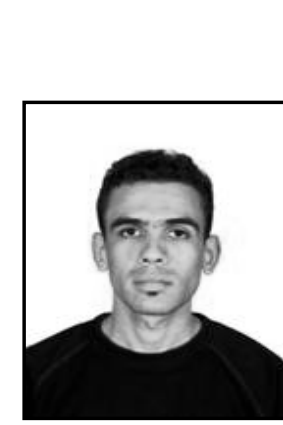

\section{Authors}

Ali Medjebouri was born in Skikda, Algeria in 1984. He received his Engineer degree in Automatic Control Systems in 2007 and his magister degree in Mechatronics in 2010 from the University of 20 August 1955, Skikda, Algeria. Currently, he is a PhD student. His research interests concern robotics and system's control.

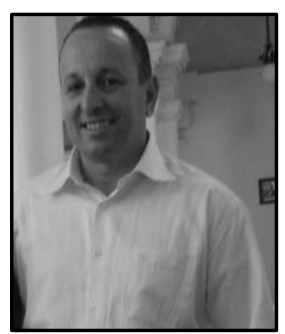

Lamine Mehennaoui was born in Constantine,Algeria, in 1967. He received the Engineer Degree in 1990, the Magister. Degree in 1994 and the PhD. Degree in 2006 all in Automatic Control Systems from the University of Baji Mokhtar, Annaba, Algeria. Since 2003, he was an affiliated researcher in the laboratory Automatic Skikda. Currently he is assistant professor in the Electrical Engineering Department of the University of 20 August 1955, Skikda Algeria. His research interests concern robotics and system's control. 